\title{
To Study The Effect Of Center Frequency And Bandwidth On Quality Factor Of Microstrip Interdigital Bandpass Filter.
}

\author{
${ }^{1}$ Abhishek Kumar Tripathi, ${ }^{2}$ Anil Kumar, ${ }^{3}$ Santosh Tripathi, ${ }^{4}$ A.K Jaiswal \\ Shiats, Nani, Allahabad
}

Abstract: In this paper interdigital bandpass filter with under coupled quarter wavelength resonator is proposed. In this we studied the quality factor of interdigital bandpass filter and showed the effect of center frequency and bandwidth on $Q$-value. $Q$ is proportional to a dominant resonator dimension and can be sensitive to manufacturing processes as well.

\section{Introduction}

Bandpass filter is a device that can be used in both transmitter and receiver in several communication systems, such as mobile phones, terrestrial network, satellite communication and many other applications. The interdigital filters have number of attractive features:

1. They are very compact.

2. The tolerance required in their manufacturing are relatively relaxed as a result of relatively large spacing between resonator elements.

3. The second passband is centered at three times the center frequency of first passband and there is no possibility of spurious response in between.

4. The rate of cutoff and the strength of the stopbands are enhanced by multiple-order poles of attenuation at DC and at even multiples of center frequency of the first passband.

5. These filters can be fabricated in structural forms which are self-supporting so that dielectric material need not be used. Thus dielectric loss ca be eliminated.

In this paper we studied the quality factor of interdigital filter and showed the effect of center frequency, fo and bandwidth, BW on quality factor.
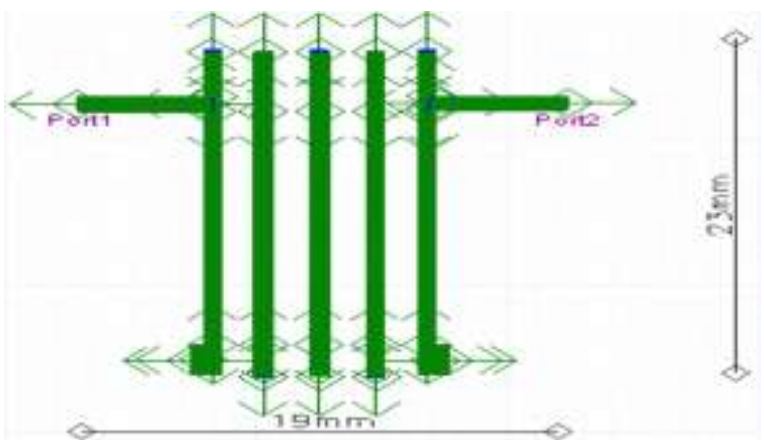

Figure 1. The EM model of bandpass filter

II.

System Analysis

Most microwave system consists of many active and passive components that are difficult to design and manufacture with precise frequency characteristics. In contrast, microwave passive filters can be designed and manufactured with remarkably predictable performance. As a result, microwave systems are usually designed so that all the troublesome components are relatively wide in frequency response to minimize their effect on the overall system.

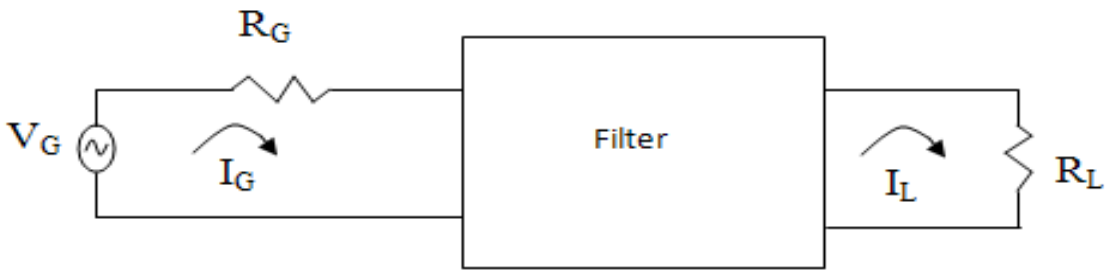

Figure 2. General form of a filter network 
In a later paper, Dishal outlined a design procedure for a tapped interdigital filter using the fo, $\mathrm{Kr}, \mathrm{r}+1$ and $\mathrm{Q}$ parameter. Once the required K and Q are known, Dishal's method was measured experimental hardware to generate a design curve for each parameter.

The filter outside terminal Q value and the inter-resonator coupling coefficient $\mathrm{K}$ were used to seek the microstrip filter parameters and calculated by:

$$
\begin{aligned}
& K_{i, i+1}=\frac{B W}{f o \sqrt{g_{i} g_{i+1}}} \\
& \backslash Q=\frac{f_{o} g_{i}}{B ~ W}
\end{aligned}
$$

Where, BW is the filter passband band width $\mathrm{g}_{\mathrm{i}}$ is the passband prototype.

At first we take bandwidth of the filter constant $200 \mathrm{MHZ}$ and vary the center frequency from $1 \mathrm{GHZ}$ to 3.5GHZ, g1= 1.3394, we see that response of quality factor. Again we take our center frequency fo constant $1.3 \mathrm{GHZ}$ and vary the band width $100 \mathrm{MZ}$ to $280 \mathrm{MHZ}$ and observe the response of quality factor.

$\mathrm{Q}$ is proportional to a dominant resonator dimension and can be sensitive to manufacturing processes as well. Insertion loss in the passband is also proportional to time delay and will increase at band edges.

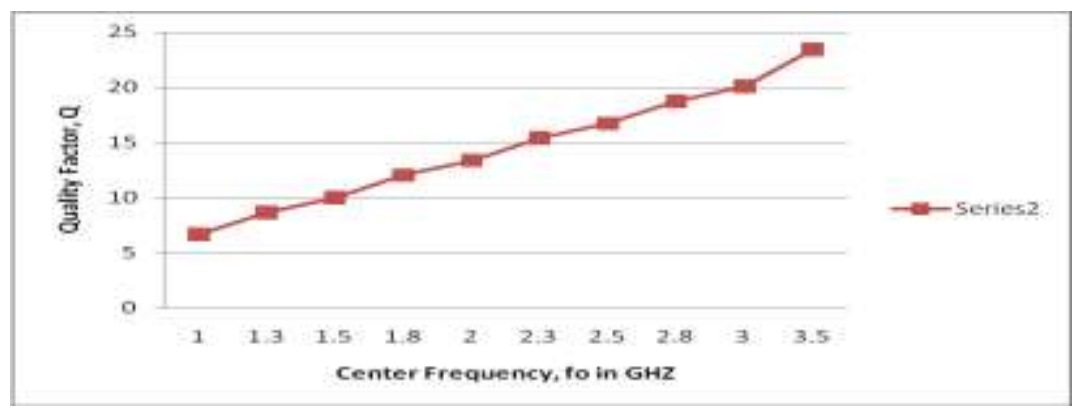

Figure 3.

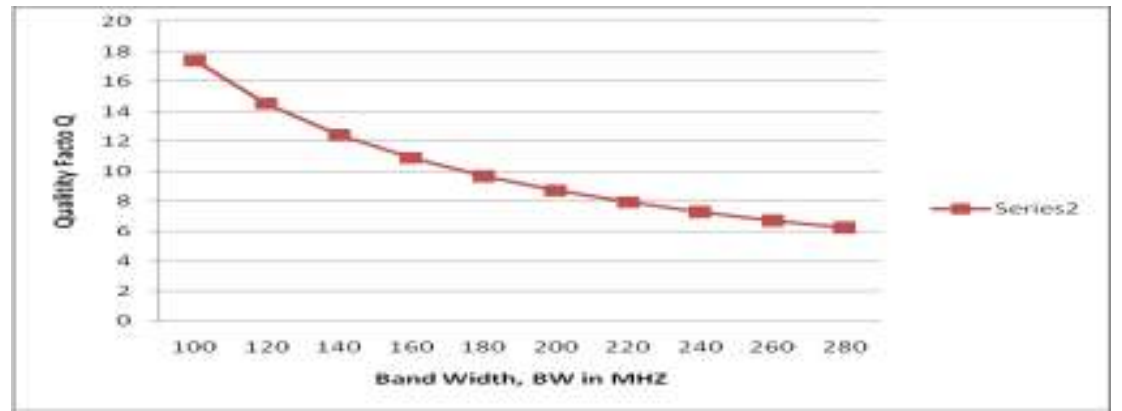

Figure 4.

III.

Result

In this report as we have seen that on increasing the center frequency, taking BW constant the Q-value of filter increases linearly. When we take fo. Constant and increase bandwidth the Q-value decreases.

\section{Conclusion}

A novel interdigital bandpass filter Q-value can be optimize by taking center frequency, fo $2.4 \mathrm{GHZ}$ and BW 200MHZ, because on increasing the center frequency bandwidth also get increases at 2.4GHZ center frequency BW of $200 \mathrm{MHZ}$ can be obtained.

\section{References}

[1] Ku Jia, Yu Na, Shi Bingxia, and Zhao "The miniaturization designs of mictrostrip interdigital bandpass filter". The deprtment of Electronics Information Engineering, Hebei University of Technology, Tiannjin. $2^{\text {nd }}$ International conference on power Electronics \& intelligent Transportation system 2009.

[2] Daniel G. Swanson, Jr. "Narrow- band microwave filter design". Tyco Electronics Lowell M.A

[3] George L. Matthaei "Interdigital Band-Pass Filters" IRE Transactions on Microwave Theory and Techniques, November 1962

[4] George L. Matthaei "MicrowaveFilters, Impedance Matching Networks and Coupling Structure" 